\title{
Agenesis of the Corpus Callosum
}

National Institute of Neurological Disorders and Stroke (NINDS)

\section{Source}

National Institute of Neurological Disorders and Stroke (NINDS). Agenesis of the Corpus

Callosum Information Page.

Agenesis of the corpus callosum (ACC) is one of several disorders of the corpus callosum, the structure that connects the two hemispheres (left and right) of the brain. In ACC the corpus callosum is partially or completely absent. It is caused by a disruption of brain cell migration during fetal development. ACC can occur as an isolated condition or in combination with other cerebral abnormalities, including Arnold-Chiari malformation, Dandy-Walker syndrome, schizencephaly (clefts or deep divisions in brain tissue), and holoprosencephaly (failure of the forebrain to divide into lobes.) Girls may have a genderspecific condition called Aicardi syndrome, which causes severe cognitive impairment and developmental delays, seizures, abnormalities in the vertebra of the spine, and lesions on the retina of the eye. ACC can also be associated with malformations in other parts of the body, such as midline facial defects. The effects of the disorder range from subtle or mild to severe, depending on associated brain abnormalities. Children with the most severe brain malformations may have intellectual impairment, seizures, hydrocephalus, and spasticity. Other disorders of the corpus callosum include dysgenesis, in which the corpus callosum is developed in a malformed or incomplete way, and hypoplasia, in which the corpus callosum is thinner than usual. Individuals with these disorders have a higher risk of hearing deficits and cardiac abnormalities than individuals with the normal structure. Impairments in social interaction and communication in individuals having a disorder of the corpus callosum may overlap with autism spectrum disorder behaviors. It is estimated that at least one in 4,000 individuals has a disorder of the corpus callosum. 